\title{
Desempenho térmico de motores elétricos aletados: solução bidimensional pelo método de separação de variáveis
}

\author{
Thermal performance of finned electric motors: two-dimensional \\ solution by separation of variables technique
}

1 Tatiana Almeida Mota Santos tattimota@gmail.com

2 Élcio Nogueira

1 Centro Universitário de Volta Redonda - UniFOA.

2 Centro Universitário de Volta Redonda - UniFOA/AEDB/UERJ

\section{RESUMO}

O motor elétrico é uma máquina que se destina a transformar energia elétrica em energia mecânica. Na indústria, é o mais usado de todos os tipos de motores, pois combina vantagens, como utilização de energia elétrica, construção simples, facilidade de transporte, baixo custo, grande versatilidade de adaptação às cargas dos mais diversos tipos com melhores rendimentos. A potência útil fornecida pela máquina elétrica na ponta do eixo é menor que a potência absorvida da linha de alimentação. A diferença entre essas duas potências representa a perda que, transformada em calor, aquece excessivamente o motor elétrico e pode ter como consequência a sua queima. Para a dissipação do calor, faz-se necessário à utilização de aletas, facilitando a troca de calor do motor elétrico com o meio que o circunda. Neste trabalho, desenvolveu-se uma solução teórica aproximada, bidimensional, através da Técnica de Separação de Variáveis, supondo temperatura prescrita na base da aleta de perfil retangular. Foram obtidos resultados para eficácia e eficiência, para a aleta e para o motor, que foram comparados com resultados de modelos teóricos unidimensionais exatos. Nas simulações efetuadas, o coeficiente de transferência de calor por convecção externo, principal responsável pelo desempenho térmico do motor, varia em ampla faixa de valores, com o objetivo de demonstrar a consistência do modelo desenvolvido. Em síntese, trata-se de um esforço adicional na busca de solução teórica realística para o desempenho térmico de motores elétricos. Dois tipos de materiais para confecção da carcaça do motor foram utilizados para análise: alumínio e ferro fundido.

\section{Palavras-Chave:}

Motores elétricos. Desempenho térmico. Aletas. Alumínio e ferro fundido.

\begin{abstract}
The electric motor is a machine that is intended to transform electrical energy into mechanical energy. It is the most used of all types of engines, as it combines advantages such as the use of electric power, simple construction, ease of transport, low cost, great versatility to adapt to loads of the most diverse types with better income. The usable power supplied by the electrical machine at the tip of the shaft is less than the absorbed power of the power line. The difference between these two powers represents loss, which transformed into heat excessively warms the electric motor and may have, as a consequence, its burning. For heat dissipation, it is necessary to use fins, facilitating the exchange of heat of the electric motor with the medium that surrounds it. In this work, an approximate, two-dimensional theoretical solution was developed, using the variable separation technique, assuming the prescribed temperature at the base of the rectangular profile fin. Results were obtained for efficacy and efficiency, for the fin and for the motor, and were compared with results of exact one-dimensional theoretical models. In the simulations performed the external convection heat transfer coefficient, the main responsible for the thermal performance of the motor, varies in a wide range of values, with the objective of demonstrating the consistency of the developed model. In summary, this is an additional effort in the search for a realistic theoretical solution for the thermal performance of electric motors. Two types of materials for the manufacture of the motor housing were used for analysis: aluminum and cast iron.
\end{abstract}

\section{Keywords:}

Electric motors. Thermal performance. Fins. Aluminum and cast iron.

\section{Como você deve citar?}

SANTOS, Tatiana Almeida Mota; NOGUEIRA, Élcio Nogueira. Desempenho térmico de motores elétricos aletados: solução bidimensional pelo método de separação de variáveis. Cadernos UniFOA, Volta Redonda, n. 38, p. 37-50, dez. 2018. 
Desempenho térmico de motores elétricos aletados:

solução bidimensional pelo método de separação de variáveis

\section{INTRODUÇÃO}

O motor elétrico é o responsável pela transformação da energia elétrica em mecânica, principal forma de uso final da energia elétrica na indústria. Sua aplicação combina vantagens como utilização de energia elétrica, construção simples, facilidade de transporte, baixo custo e grande versatilidade de adaptação às cargas do motor.

A diferença entre a potência útil fornecida pelo motor na ponta do eixo e a potência absorvida na linha de alimentação representa a perda do sistema, e essa energia é transformada em calor. 0 calor produzido deve ser eficientemente dissipado para evitar o aquecimento excessivo do motor e sua consequente queima.

A troca de calor no motor é diretamente proporcional a uma ventilação eficiente, a diferença de temperatura entre a superfície da carcaça e do meio que a circunda, da área total de troca da carcaça e das aletas instaladas. Nos motores elétricos, é comum o uso de superfícies estendidas, denominadas aletas, que aumentam a área de troca como mecanismo de otimização da transferência de calor. A condutividade térmica do material da aleta tem forte efeito na distribuição de temperatura ao longo dela, afetando o grau no qual a taxa de transferência de calor é aumentada ou diminuída. Assim como o material, a geometria também influencia na área disponível para troca.

Os motores elétricos podem apresentar variações, pelo fato de serem submetidos a esforços elétricos e mecânicos distintos, às instalações em diferentes tipos de ambiente e temperatura, e por nem sempre serem submetidos à manutenção, limpeza e lubrificação ideal, ainda que trabalhem com a mesma potência. Tais fatores permitem que esses motores percam, aproximadamente, 3 a $4 \%$ do rendimento durante sua vida útil (CARDOSO et al., 2009).

Estudos sobre esses equipamentos são realizados com frequência, buscando economia de energia, reduzindo seu peso e elevando o rendimento. No entanto, ainda é comum a queima de motores elétricos, devido a elevadas temperaturas de operação a qual são submetidos, nos mais diversos tipos de ambientes industriais em que se encontram. É imprescindível a identificação, análise e correção de problemas, seja de ordem mecânica ou elétrica, de modo a aumentar o nível de confiabilidade do sistema e os lucros operacionais, uma vez que a queima de um motor implica em sua substituição, comprometendo horas ou até dias de produção, gerando custos à empresa.

Em função da importância do que foi argumentado no parágrafo acima, trabalhos teóricos e experimentais têm sido desenvolvidos, dentre eles, destacam-se: a Tese de Doutorado de Christopher Beng (2006) e a Dissertação de Mestrado e Tese de Doutorado de Voigdlener (2004; 2010).

Beng (2006) realizou um estudo teórico-experimental, "tridimensional", utilizando CFD (Fluidodinâmica Computacional), DNS [Simulação Numérica Direta, com modelos de turbulência algébrico e diferencial $(\mathrm{K}$ e E $)$ ]. Durante o desenvolvimento da tese, foram utilizados computadores de alto desempenho, com custo altíssimo, para geração de dados.

Voigdlener (2010), no Programa de Pós-Graduação em Engenharia Mecânica da Universidade Federal de Santa Catarina, desenvolveu um modelo térmico com parâmetros acoplados, que consiste na combinação de parâmetros térmicos globais e de parâmetros térmicos distribuídos em diferentes componentes do motor (carcaça, rotor e estator). A principal constatação na aplicação do modelo desenvolvido por Voigdlener (2010), após uma análise de sensibilidade, é que o parâmetro mais crítico no resfriamento do motor é o coeficiente convectivo de calor em sua superfície externa. 
Além da análise teórica, Voiglener (2010) realizou medições experimentais no coeficiente de transferência de calor convectivo local da carcaça do motor para o ar ambiente, explorando não uniformidades e seu impacto na temperatura global do motor. Além disso, foram efetuados ensaios em rotações de 900 rpm, $1200 \mathrm{rpm}$ e $1800 \mathrm{rpm}$, com o objetivo de se mensurar o efeito da rotação no coeficiente de transferência de calor externo.

Outras duas análises, de ordem teórica e unidimensional exata, foram publicadas por Novais e Comitra (2014) que exploraram a condição de fluxo prescrito na base da aleta. Soares (2015) analisou o efeito da resistência térmica entre o estator e a carcaça para obtenção do fluxo de calor na base da aleta. Os resultados dos modelos unidimensionais exatos citados foram surpreendentes, apesar das limitações inerentes a esse tipo de abordagem teórica, uma vez que os valores obtidos para o coeficiente de transferência de calor externo coincidiram com valores determinados por Beng (2006), dentro da faixa de erro experimental definido para esse parâmetro.

\section{OBJETIVO}

O objetivo do presente trabalho é realizar uma análise teórica aproximada, através da Técnica de Separação de Variáveis, do desempenho térmico em motores aletados, utilizando sistemas de aletas com perfis retangulares e condição de temperatura prescrita na base (carcaça do motor), com determinação de uma solução analítica bidimensional aproximada para perfis de temperatura, taxa de transferência de calor, eficiência e efetividade para as aletas e para o motor, com solução do problema de autovalor associado. Pretende-se contribuir para estudos futuros, na busca de uma solução tridimensional exata e na determinação de uma solução viável e compatível com a prática, que reduza a "queima" e consequente perda de motores elétricos no âmbito industrial.

\section{METODOLOGIA}

De acordo com Incropera (2008), são utilizados dois parâmetros para mensurar o desempenho de uma aleta: a eficiência e a efetividade (eficácia). A eficiência é mensurada através do quociente entre a taxa de transferência de calor da aleta e a taxa de transferência de calor, se a temperatura dela for constante, considerando essa temperatura igual à da base, podendo ser descrita pela equação a seguir. A eficiência é apresentada em termos percentuais, conforme Equação 01.

$$
\eta_{\text {aleta }}=\frac{q_{\text {real }}}{h_{2} \times A_{T_{\text {aleta }}} \times \theta_{b}}
$$

Já a eficácia ou efetividade da aleta é a razão entre a taxa de transferência de calor da aleta e a taxa de transferência de calor da superfície analisada sem a aleta. É formulada conforme Equação 02 abaixo.

$$
\text { eff } f_{\text {aleta }}=\frac{q_{\text {comaleta }}}{h_{2} \times A_{b} \times \theta_{b}}
$$

Onde:

$$
\begin{aligned}
& A_{T_{\text {aleta }}} \rightarrow \text { Área total de troca da aleta ou área superficial da aleta } \\
& A_{b} \rightarrow \text { Área da base da aleta }\left(A_{b}=b b . L\right)
\end{aligned}
$$




$$
\begin{aligned}
& \dot{q}_{\text {aleta }} \rightarrow \text { Taxa de calor trocada por cada aleta } \\
& \theta_{b} \rightarrow \text { Temperatura auxiliar na base da aleta }\left(\theta_{b}=T_{b}-T_{\infty}\right) \\
& h_{2} \rightarrow \text { Coeficiente de convecção externo }
\end{aligned}
$$

Para calcular a eficiência do motor elétrico aletado, usado na pesquisa, utilizamos as seguintes fórmulas:

$$
\eta_{\text {motor }}=\frac{32 \cdot \dot{q}_{\text {aleta }}}{h \cdot\left[A_{L}+\left(A_{\text {motor }}-32 \cdot A_{b}\right)\right] \cdot\left(T_{b}-T_{\infty}\right)}
$$

Como o rendimento do motor é a razão entre duas grandezar nn mnnma unidade, seu resultado é adimensional e expresso em forma de porcentagem, ou seja, $0 \leq \eta_{\text {motor }} \leq 1$.

Não se pode negar a importância do motor elétrico no âmbito industrial. É fundamental que ocorra dissipação adequada da energia térmica gerada no equipamento, de forma a evitar seu aquecimento excessivo e consequente queima e inutilização. E para que isso ocorra, é necessário que uma ventilação eficiente seja aplicada ao sistema. A ventilação depende não só da transferência de calor por convecção a qual o sistema está submetido, já que essa troca ocorre entre a superfície do motor e um fluído em movimento (ar), que se encontra sob diferentes temperaturas, mas também a condução aliada à convecção na região interna. Há uma resistência térmica gerada na interface de contato entre a parede do motor e seu isolamento, devido ao contato imperfeito entre os meios, como pequenas rugosidades e ondulações. Nessa região, ocorre condução nos pontos de contato e condução através dos fluidos aprisionados nos interstícios dessas imperfeições.

Segundo Ordenes (2008), "o coeficiente de convecção é uma constante de proporcionalidade que leva em consideração a geometria da superfície, a natureza do escoamento, as propriedades e velocidade do fluído, a condutividade térmica do material envolvido, o acabamento superficial, a dureza, a temperatura média na interface e o fluxo de calor".

Moreira (2012) afirma que a determinação do coeficiente de convecção torna-se complexa e exige solucionar equações diferenciais da condução de calor, diante de tantas variáveis envolvidas.

Sendo assim, é preciso determinar dois coeficientes de convecção, um referente à região externa e outro à região interna da carcaça do motor. Para a superfície interna, deverá ser levado em consideração as imperfeições macroscópicas existentes na mesma e a pressão de contato dos materiais, o fluido contido nos interstícios, o filme de óxidos aplicado a essa superfície e o calço metálico envolvido. Portanto, esse último coeficiente de convecção é o mais complexo dos dois processos, e o mais difícil de se obter experimentalmente (NOVAIS; COMITRA, 2014).

Nesse contexto, obteve-se uma solução analítica aproximada da equação diferencial parcial, necessária à determinação do perfil de temperatura e da taxa de transferência de calor que ocorre em aletas de ferro fundido dispostas na carcaça de um motor elétrico, esquematicamente representado na Figura 01 abaixo: 
Figura 01 - Representação esquemática da geometria do problema

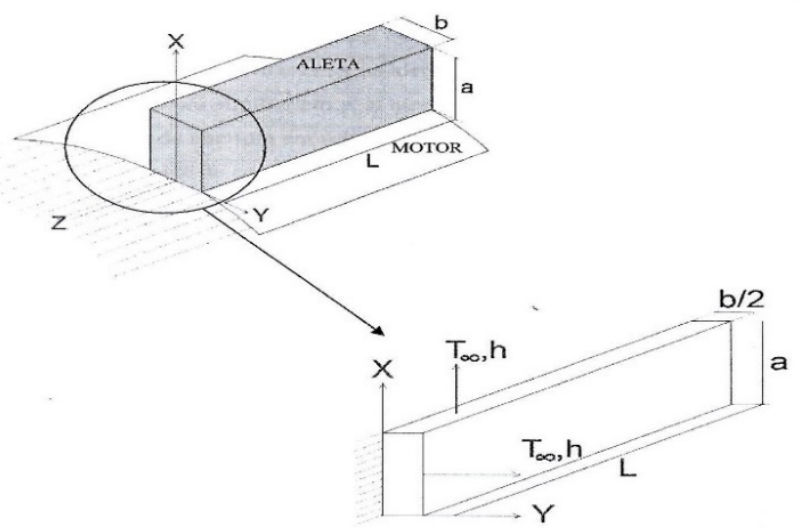

Fonte: dos autores, 2018.

Representa-se o problema acima definido através de uma Equação Diferencial Parcial (EDP), descrita pela equação abaixo, com condição de regime permanente como hipótese:

$\frac{\partial^{2} T(x, y)}{\partial x^{2}}+\frac{\partial^{2} T(x, y)}{\partial y^{2}}=\frac{h \cdot A_{\text {troca }}}{k \cdot \text { Vol }} \cdot[T(x, y)-T \infty]$

Definindo-se:

$\theta(x, y)=\frac{T(x, y)-T \infty}{T_{M}-T_{\infty}}$

$\frac{\partial^{2} \theta(x, y)}{\partial x^{2}}+\frac{\partial^{2} \theta(x, y)}{\partial y^{2}}=\frac{h \cdot A_{\text {troca }}}{k \cdot V o l} \cdot(\theta(x, y))$

Pela hipótese de separação de variáveis:

$\theta(x, y)=\theta_{1}(x) \cdot \theta_{2}(y)$

$\frac{1}{\theta_{1}(x)} \cdot \frac{\partial^{2} \theta_{1}(x)}{\partial x^{2}}-\frac{h \cdot A_{L}(x)}{k \cdot V o l}=-\frac{1}{\theta_{2}(y)} \cdot \frac{\partial^{2} \theta_{2}(y)}{\partial y^{2}}+\frac{h \cdot A_{L}(y)}{k \cdot V o l}=-\omega^{2}$

Onde:

$A_{\text {troca }}=A_{L}(x)+A_{L}(y) ; \mathrm{Vol}=A_{B} . L ; P_{B}=2\left(L+\frac{b b}{2}\right)$

Assim, tem-se que:

$$
\begin{aligned}
& \frac{d^{2} \theta_{1}(x)}{d x^{2}}-\left[\frac{h \cdot A_{\text {troca }}}{k \cdot V o l}-\omega^{2}\right] \cdot \theta_{1}(x)=0 \quad \operatorname{com} A_{L}(x) \gg A_{L}(y) \\
& \frac{d^{2} \theta_{2}(y)}{d y^{2}}+\left[\omega^{2}-\frac{h \cdot A_{L}(y)}{k \cdot V o l}\right] \theta_{2}(y)=0
\end{aligned}
$$


Condições de contorno em x:

$$
\begin{aligned}
& \mathrm{x}=0 \quad \rightarrow \quad \theta_{1}(0)=\theta_{b} \quad \rightarrow \quad \theta_{b}=1 \\
& \mathrm{x}=\mathrm{a} \rightarrow-k \cdot \frac{d \theta_{1}(x)}{d x}=h \cdot \theta_{1}(a)
\end{aligned}
$$

Condições de contorno em y:

$$
\begin{aligned}
& \mathrm{y}=0 \rightarrow \frac{d \theta_{2}(y)}{d y}=0 \\
& \mathrm{y}=b / 2 \rightarrow-k \cdot \frac{d \theta_{2}(y)}{d y}=h \cdot \theta_{2}(b / 2)
\end{aligned}
$$

Tem-se como solução geral em x:

$$
\theta_{1}(x)=C_{1} \cdot \cosh (m \cdot x)+C_{2} \cdot \operatorname{senh}(m \cdot x)
$$

onde,

$$
\begin{aligned}
C_{1} & =\theta_{b} \\
C_{2} & =-\theta_{b} \cdot\left[\frac{\cosh (m \cdot a)+\frac{k \cdot m}{h} \cdot \operatorname{senh}(m \cdot a)}{\frac{k \cdot m}{h} \cdot \cosh (m \cdot a)+\operatorname{senh}(m \cdot a)}\right]
\end{aligned}
$$

Temos como solução geral em y:

$\theta_{2}(y)=C_{3} \cdot \cos (\omega(n) \cdot y)+C_{4} \cdot \operatorname{sen}(\omega(n) \cdot y)$

onde,

$$
C_{4}=0
$$

$C_{3} \cdot \frac{k \cdot \omega_{2}}{h} \cdot \operatorname{sen}(\omega \cdot b / 2)=C_{3} \cdot \cos (\omega \cdot b / 2)$

Logo:

$$
\begin{aligned}
& \omega \cdot \operatorname{tg}(\omega \cdot b / 2)=\frac{h}{k} \\
& \omega_{2}=\omega \cdot b / 2 \\
& \omega=\frac{\omega_{2}}{b b}
\end{aligned}
$$

$\omega_{2} \cdot \operatorname{tg}\left(\omega_{2}\right)=\frac{h \cdot b b}{k}$ 
Como

$\theta(x, y)=\theta_{1}(x) \cdot \theta_{2}(y)$

Tem-se como solução geral do problema:

$\theta(x, y)=\sum_{n=0}^{\infty}\left\{\theta_{b} \cdot \cosh (m \cdot x)-\theta_{b} \cdot\left[\frac{\cosh (m \cdot a)+\frac{k \cdot m}{h} \cdot \operatorname{senh}(m \cdot a)}{\frac{k \cdot m}{h} \cdot \cosh (m \cdot a)+\operatorname{senh}(m \cdot a)}\right] \cdot \operatorname{senh}(m \cdot x)\right\} C_{3} \cdot \cos (\omega(n) \cdot y)$

onde,

$m=\sqrt{\frac{h \cdot P_{B}}{k \cdot A_{b}}-\omega^{2}}$

e,

$C_{3}=\frac{2}{1+|\cos (\omega(n) \cdot b b)|}$

A Equação 14 representa o problema de autovalor associado, cuja solução foi obtida pelo método da bissecção. Os primeiros 06 autovalores encontram-se representados na Tabela 01 abaixo:

Tabela 01 - Primeiros seis autovalores - Equação 15

$\boldsymbol{h b} \boldsymbol{b} / \boldsymbol{k}$

$\omega_{21}$

$\omega_{22}$

$\omega_{23}$

$\omega_{24}$

$\omega_{25}$

$\omega_{26}$

\begin{tabular}{|l|c|c|c|c|c|c|}
\hline 0.101 & 0.3125 & 3.1734 & 6.2992 & 9.4354 & 12.5744 & 15.7143 \\
\hline 0.201 & 0.4338 & 3.2042 & 6.315 & 9.446 & 12.5823 & 15.7207 \\
\hline 0.301 & 0.5226 & 3.2344 & 6.3307 & 9.4566 & 12.5903 & 15.7271 \\
\hline 0.401 & 0.5939 & 3.2638 & 6.3463 & 9.4671 & 12.5982 & 15.7334 \\
\hline 0.501 & 0.6538 & 3.2926 & 6.3618 & 9.4776 & 12.6061 & 15.7398 \\
\hline 0.601 & 0.7055 & 3.3206 & 6.3771 & 9.488 & 12.614 & 15.7461 \\
\hline 0.701 & 0.751 & 3.348 & 6.3924 & 9.4984 & 12.6218 & 15.7524 \\
\hline 0.801 & 0.7914 & 3.3746 & 6.4075 & 9.5088 & 12.6297 & 15.7587 \\
\hline 0.901 & 0.8277 & 3.4006 & 6.4226 & 9.5191 & 12.6375 & 15.7650 \\
\hline
\end{tabular}

Fonte: dos autores, 2018.

Para determinação da taxa de transferência de calor através da aleta:

$$
\dot{q}=\sum_{n=0}^{\infty} k \cdot A_{b} \cdot m \cdot \theta_{b} \frac{C_{3}(n)}{\omega(n)} \cdot \operatorname{sen}(\omega(n) \cdot b b)
$$

O primeiro autovalor é dominante e representa uma boa aproximação para a taxa de transferência de calor, para $C \neq 0$ :

$$
q_{\text {aprox }}^{\cdot}=k \cdot A_{b} \cdot m \cdot \theta_{b} \frac{C_{3}(0)}{\omega(0)} \cdot \operatorname{sen}(\omega(0) \cdot b b)
$$


Para uma solução aproximada, compatível com a solução unidimensional clássica, adotou-se:

$$
m^{2}=\frac{h \cdot P_{b}}{k A_{b}}-\omega^{2} \text { e } A_{L}(y)=0 ; A_{L}(x)=P_{b} L ; \text { Vol }=A_{b} L
$$

Dessa forma, tem-se as equações aproximadas que definem a taxa de transferência de calor e o comportamento da temperatura ao longo de uma aleta no motor, considerando temperatura prescrita na sua base.

\section{RESULTADOS E DISCUSSÕES}

Os resultados obtidos são referentes à solução analítica aproximada para a temperatura prescrita na base de apenas uma das trinta e duas aletas existentes na carcaça do motor, descrevendo-se o comportamento da aleta, quando constituída de ferro fundido $\left(k=80 \mathrm{~W} / \mathrm{m}^{2} . K\right)$, assim como, quando é composta de alumínio $\left(k=237 \mathrm{~W} / \mathrm{m}^{2} . K\right)$.

$\mathrm{Na}$ Figura 02, a seguir, temos a representação da distribuição de temperatura ao longo da coordenada $\mathrm{x}$, considerando solução unidimensional em aletas de ferro fundido, quando submetidas a coeficientes de transferência de calor por convecção externo (h) variando.

Figura 02 - Solução ao longo da coordenada x com coeficiente de transferência de calor por convecção variando.

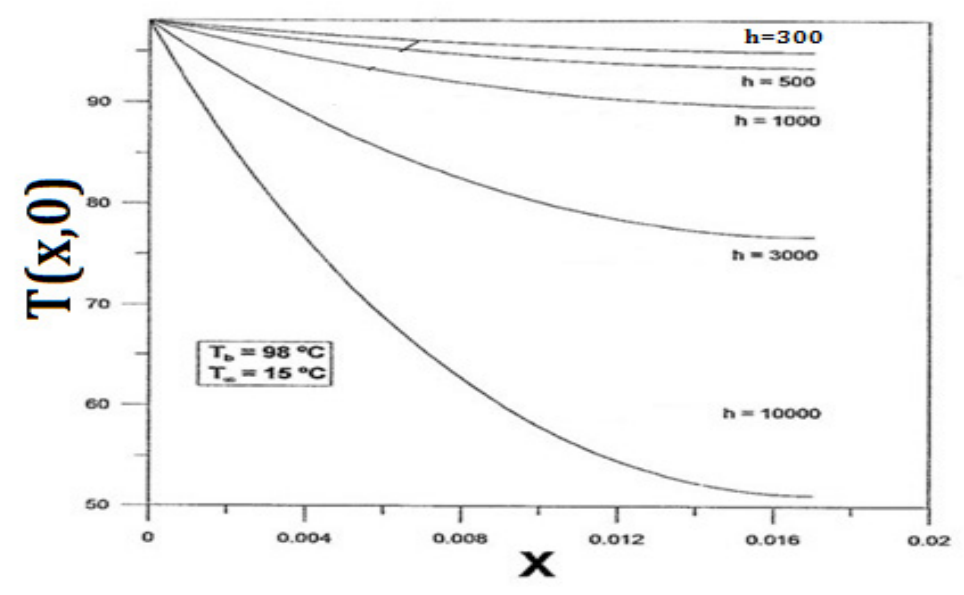

Fonte: dos autores, 2018.

$\mathrm{Na}$ Figura 02, a análise foi efetuada, considerando-se um coeficiente de transferência de calor (h) variando da ordem de $3.10^{2}$ a $10^{4}\left(\mathrm{~W} / \mathrm{m}^{2} . \mathrm{K}\right)$. Assim, foi possível visualizar que, com um coeficiente de transferência de calor baixo, a taxa de transferência de calor será baixa e insuficiente para a retirada de calor necessária ao sistema, mantendo a temperatura bem próxima à temperatura da base em todo o eixo x. Percebe-se que a medida que há um aumento no coeficiente de transferência de calor, aumenta-se a troca de calor, onde a temperatura ao longo do eixo $x$ tende a cair, aproximando-se da temperatura do meio na extremidade. A temperatura na base, $x=0$, mantém-se intacta, uma vez que é uma das condições de contorno do problema. Essa situação é compatível com a solução de uma aleta unidimensional, conforme Novaes e Comitra $(2014,2017)$ e Soares $(2015)$. 
A Figura 03 exibe a eficácia na aleta, quando submetida a um h entre $10^{2}$ e $10^{3}\left(\mathrm{~W} / \mathrm{m}^{2} . \mathrm{K}\right)$. Há uma significativa alteração no valor da eficácia, quando se compara os modelos unidimensional e bidimensional. De fato, essa alteração é mais pronunciada no caso de aleta de alumínio, onde a faixa de eficácia>2 é amplamente estendida para valores de $\mathrm{h}$. Esse fato demonstra que o modelo unidimensional subestima a troca de calor para valores de $\mathrm{h}$ mais elevados, o que limita a utilização de aletas nessa faixa. Como estamos trabalhando com variáveis não adimensionais, os resultados necessitam ser mais bem analisados, com variações dos parâmetros físicos que modulam a solução. Uma tal análise, aqui, é inviável, uma vez que são muitos parâmetros a serem analisados.

Figura 03 - Comparações para a Eficácia da aleta de alumínio e de ferro fundido (Unidimensional x Bidimensional).

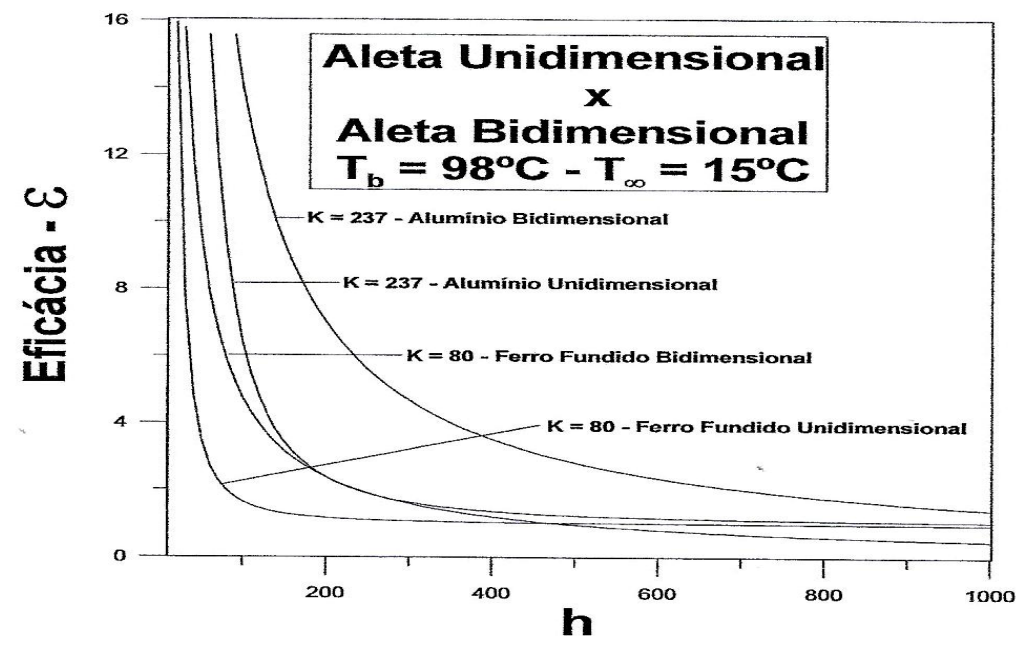

Fonte: dos autores, 2018.

Figura 04 - Desempenho da aleta bidimensional de alumínio comparada a de ferro fundido.

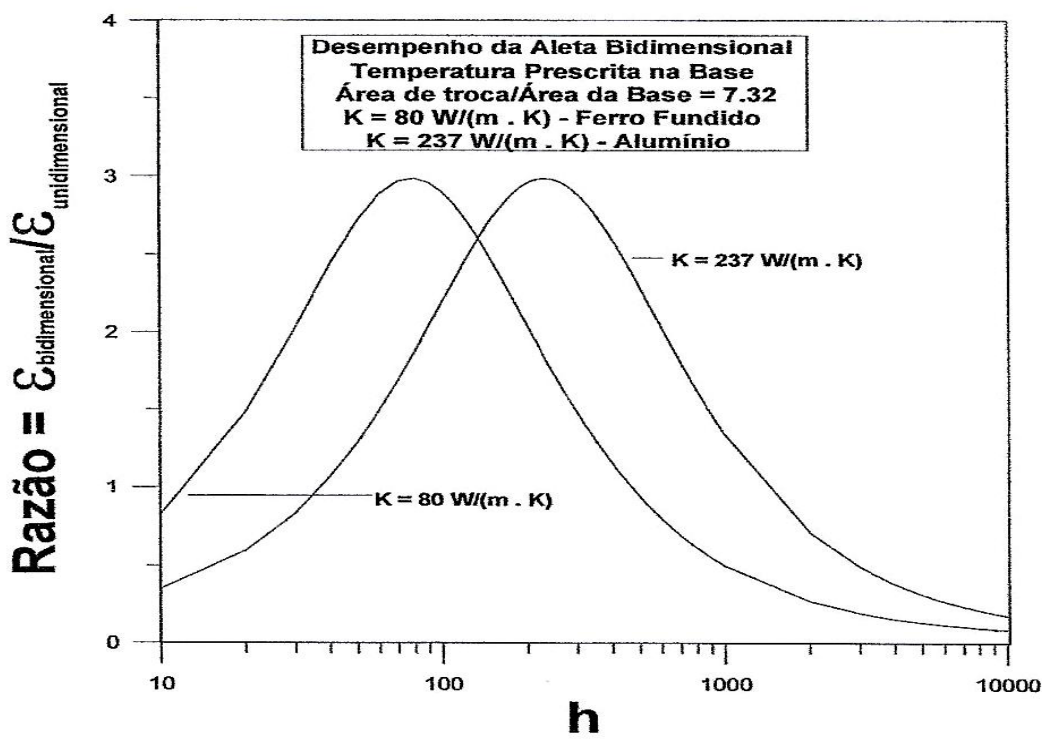

Fonte: dos autores, 2018 
A Figura 04 demonstra que o modelo desenvolvido para aleta bidimensional prevê melhor desempenho que o modelo unidimensional, com um máximo superior igual a 3, para ambos os materiais analisados. Como já analisado anteriormente, para o alumínio, há uma ampliação na faixa de utilização do coeficiente de transferência de calor, e o ponto de máximo ocorre exatamente para 237, coeficiente de condução do material. Esse resultado é notável, uma vez que o ponto de máximo para o ferro fundido também passa por $\mathrm{k}=80$, valor de sua condutividade térmica. Fica evidente que, se o alumínio for utilizado, os valores de $\mathrm{h}$ devem ser superiores que $\mathrm{h}$ para ferro fundido, para que se tenha desempenho similar.

De forma equivalente, observa-se através da Figura 05 que a eficiência da aleta, para modelo bidimensional em relação ao modelo unidimensional, é superior em todos os casos, estendendo os valores de $h$ utilizáveis.

Figura 05 - Comparações para a Eficiência da aleta de alumínio e de ferro fundido (Unidimensional x Bidimensional).

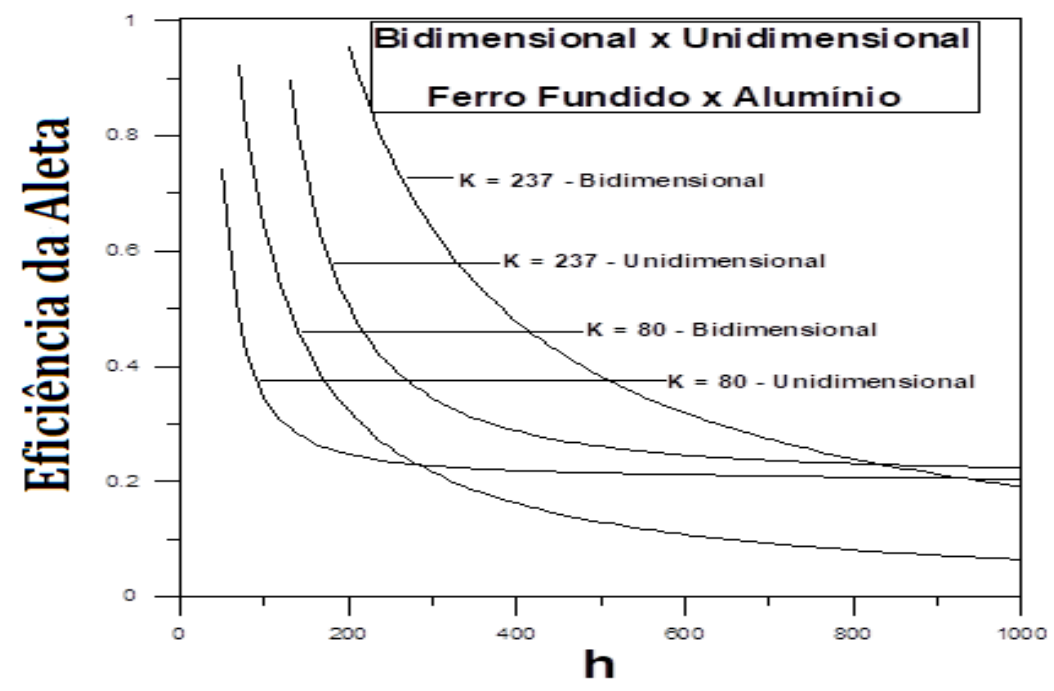

Fonte: dos autores, 2018. 
Figura 06 - Eficácia do motor de alumínio comparado ao de ferro fundido (Bidimensional x unidimensional).

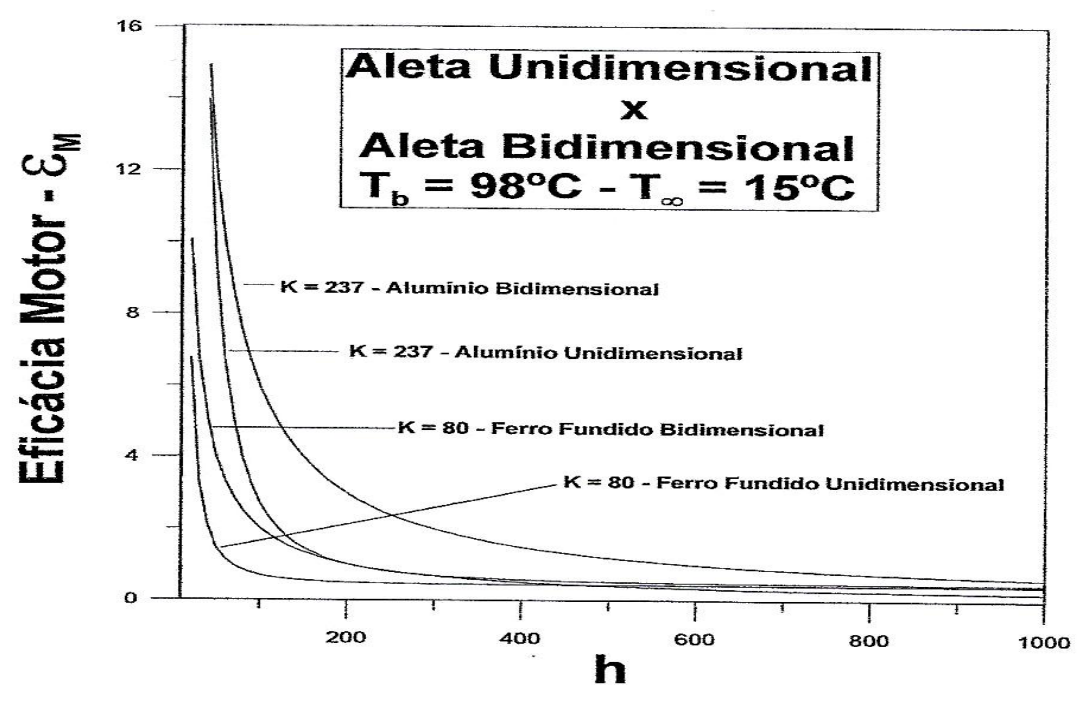

Fonte: dos autores, 2018.

De acordo com a Figura 06, a Eficácia do motor é ligeiramente inferior à Eficácia da aleta. Isso se justifica, uma vez que a área de troca com o meio é superior à área de troca das aletas.

A Figura 07 demonstra que a Eficiência do motor é ligeiramente inferior à eficiência da aleta. De forma equivalente ao mencionado para a Eficácia, a área de troca, com o motor, é ligeiramente superior à área das aletas.

Figura 07 - Eficiência do motor de alumínio comparado ao de ferro fundido (Bidimensional $\mathrm{x}$ unidimensional).

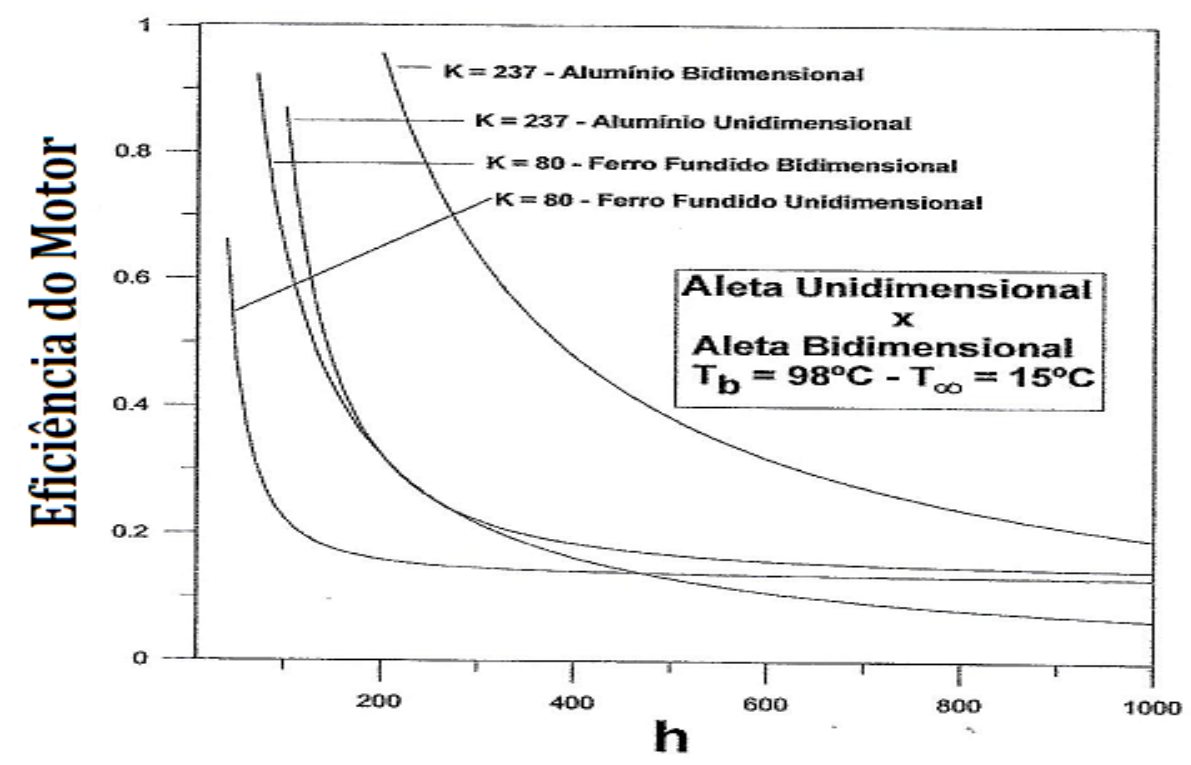

Fonte: dos autores, 2018. 
Desempenho térmico de motores elétricos aletados:

solução bidimensional pelo método de separação de variáveis

\section{CONCLUSÃO}

Os resultados permitem confirmar a importância do coeficiente de transferência de calor aplicado na região externa ao motor, de forma equivalente ao concluído por Voiglener (2010), Beng (2006), Novaes e Comitra $(2014,2017)$, Soares (2015). Quanto mais baixo o valor estabelecido para o coeficiente de transferência de calor que atua na região externa do motor, menor será a taxa de transferência de energia, não dissipando de forma eficiente o calor gerado internamente. Sendo assim, a aleta tende a permanecer na temperatura da carcaça, base das aletas, de tal maneira que essa energia térmica não dissipada acarretará no aumento da temperatura interna do motor, levando a sua "queima" e consequente inutilização. 0 coeficiente de transferência de calor tem, entretanto, limitações físicas e depende do sistema de ventilação utilizado para o motor.

As Eficiências e Eficácias se mostraram superiores para modelo bidimensional aproximado, demonstrando que a solução unidimensional subestima a troca de calor que efetivamente ocorre no processo.

Um modelo bidimensional mais complexo, exato, pode dar respostas mais efetivas sobre parâmetros importantes para o desempenho térmico do motor elétrico. Um dos aspectos fundamentais, já ressaltado, é a resistência térmica entre a carcaça e o núcleo do motor. Voiglener (2010) e Beng (2006) analisam o efeito dessa resistência, teórica e experimentalmente, no desempenho térmico do motor. 
NOMENCLATURA

\begin{tabular}{|c|c|}
\hline$T_{\infty}$ & Temperatura do meio que circunda o motor \\
\hline$T_{b}$ & Temperatura na base da aleta \\
\hline$T_{M}$ & Temperatura do motor \\
\hline$T_{L}$ & Temperatura na extremidade superior da aleta \\
\hline$a$ & Comprimento em $\mathrm{x}$ da aleta \\
\hline$b$ & Comprimento em y da aleta \\
\hline$b b=b / 2$ & Comprimento em y da aleta \\
\hline$\theta_{b}$ & Temperatura auxiliar na base da aleta \\
\hline$\theta_{1}$ & Temperatura auxiliar na variável $\mathrm{x}$ \\
\hline$\theta_{2}$ & Temperatura auxiliar na variável y \\
\hline$\alpha$ & Ângulo de abertura em relação ao centro do motor \\
\hline$h$ & Coeficiente de convecção externo \\
\hline$L$ & Comprimento do motor \\
\hline Vol. & Volume do motor \\
\hline$A_{L}$ & Área lateral de troca da aleta \\
\hline$A_{\text {troca }}$ & Área total de troca da aleta \\
\hline$A_{b}$ & Área da base da aleta \\
\hline$k$ & Condutividade térmica do material \\
\hline$P_{B}$ & Perímetro da base da aleta \\
\hline$m$ & Definição de um parâmetro \\
\hline$n$ & Definição de um parâmetro \\
\hline$\omega_{2}$ & Raiz da equação característica \\
\hline
\end{tabular}

\section{REFERÊNCIAS}

ALMEIDA, Antônio, T. L. Motores elétricos. Itajubá, MG: Universidade Federal de Itajubá, 2004.

BENG, Christopher Micallef. End Winding Cooling in Electric Machines. Thesis submitted to the University of Nottingham for the degree of Doctor of Philosophy, 2006.

CARDOSO, Rafael Balbino; NOGUEIRA, Luiz Augusto Horta; BARTONI, Edson da Costa; HADDAD, Jamil; SOUZA, Edson Palhares. Avaliação da Economia de Energia Atribuídas a Ações de Etiquetagem Energética, em Motores de Indução no Brasil. Revista Brasileira de Energia, Vol.15, N. 1, pp. 29-47, 2009.

CEZÁRIO, Cassiano A. Análise do escoamento de ar em motores de indução totalmente fechados. Florianópolis, 2007. 
Desempenho térmico de motores elétricos aletados:

solução bidimensional pelo método de separação de variáveis

DUARTE, Denise Freire; NOVAIS, Ariane Silva; NOGUEIRA, Élcio. Solução Analítica em aleta de perfil retangular. comparação de desempenho térmico entre alumínio e ferro fundido em motores elétricos. Cadernos UniFOA, Volta Redonda, 2012.

FILIPPO FILHO, G. Motor de indução. São Paulo: Érica, 2000.

INCROPERA, Frank P. et al. Fundamentos de transferência de calor e de massa. 6. ed. Rio de Janeiro: LTC, 2008.

MOREIRA, José R. Processos de transferência de calor. São Paulo: USP, 2012.

MOTA SANTOS, T. A. Análise de desempenho térmico de motores elétricos aletados: solução bidimensional em aleta retangular com temperatura prescrita na base. Trabalho de Conclusão de Curso, Engenharia Mecânica, UNIFOA, 2017.

NOVAIS, A. S.; COMITRA, R. D. F. C. Desempenho térmico de motores elétricos com fluxo de calor prescrito na base de aletas retangulares. Trabalho de Conclusão de Curso, Engenharia Mecânica, UNIFOA, 2014.

NOVAIS, A. S.; COMITRA, R. D. F. C.; NOGUEIRA, É. Análise teórica de desempenho térmico de motores de indução elétrica aletados. Cadernos UniFOA, Volta Redonda, n² 25, quadrimestral, p. 19-34, 2017.

OLIVEIRA, João Gabriel S. M. Materiais usados na construção de motores elétricos. Porto Alegre, 2009.

ORDENES M.; LAMBERT R.; GÜTHS, S. Transferência de Calor Envolvente na Edificação. Departamento de Engenharia Civil - Universidade Federal de Santa Catarina, 2008.

Soares, M. V. F. Análise da influência do coeficiente de transferência de calor por convecção na temperatura do núcleo de motor elétrico aletado com fluxo de calor prescrito na base. Trabalho de Conclusão de Curso, Engenharia Mecânica, UNIFOA, 2015.

VOIGDLENER, T. Desempenho térmico de motores elétricos de indução. Tese (doutorado) - Universidade Federal de Santa Catarina, Centro Tecnológico. Programa de Pós-Graduação em Engenharia. Florianópolis, SC, 2010.

Escoamento e transferência de calor em motores de indução. Dissertação (Mestrado em Engenharia e Ciências Térmicas) - Programa de Pós-Graduação em Engenharia Mecânica, Universidade Federal de Santa Catarina, 2004.

VOIGDLENER, T.; PRATA, A. T.; PASSOS, A. S. B. Modelo térmico híbrido com fontes de calor distribuídas em um motor de indução. Anais do XVI Congresso Brasileiro de Engenharia Mecânica, Uberlândia, 2001. 